\title{
Die Österreichische Palliativgesellschaft (OPG)
}

\author{
Wiener klinische Wochenschrift \\ The Central European Journal of Medicine
}

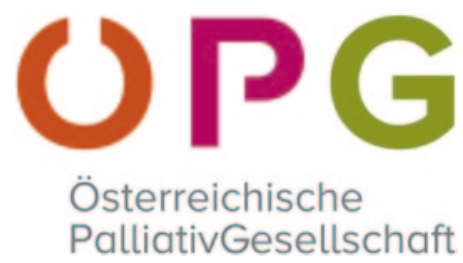

Online publiziert: 14. August 2013

(C) Springer-Verlag Wien 2013

Die Österreichische Palliativgesellschaft (OPG) ist eine multiprofessionelle wissenschaftliche Vereinigung von ÄrztInnen, Pflegenden und vieler weiterer Berufsgruppen, die mit der Betreuung schwerkranker Patienten mit fortgeschrittenem Leiden und eingeschränkter Lebenserwartung befasst sind. Sie unterstützt Initiativen in Forschung, Fortbildung und praktischer Ausübung von Palliative Care mit dem Ziel der Stärkung der einzelnen Disziplinen und ihrer Interprofessionalität. Palliative Care steht dabei für einen umfassenden Betreuungsansatz, der durch die Behandlung physischer, psychischer, sozialer und spiritueller Probleme die Lebensqualität unheilbar erkrankter schwerkranker Menschen und deren Familien verbessert.

\section{Geschichte der OPG}

Die Gründung der OPG erfolgte 1999 auf Initiative von Dr. Michaela Werni und Dr. Franz Zdrahal. Dem Gründungsvorstand gehörten neben Vertretern der wichtigsten medizinischen Fachgruppen, die Palliativpatienten betreuen, wie Onkologen, Schmerztherapeuten oder Strahlentherapeuten, auch Vertreter aller Berufsgruppen, die in Palliative Care tätig sind an, wie Pflegende, Seelsorger oder Sozialarbeiter. 2010 erfolgte unter unveränderter Beibehaltung der Multiprofessionalität der OPG eine Strukturänderung des Vorstandes. Dieser besteht nun neben den vereinsrechtlich notwendigen Funktionsträgern aus den Leitern von fünf Arbeitsgruppen, die zu den Themen Palliativpflege, Ethik, Geriatrie, Ausbildung und Sozialarbeit eingerichtet wurden.

\section{Ziele der OPG}

Die OPG versteht sich in ihrer Struktur und in ihren Aufgabenstellungen als eine interdisziplinäre wissenschaftliche Gesellschaft mit den Zielen,
- die persönlichen Kontakte unter den Mitgliedern und die Beziehungen zu den entsprechenden ausländischen Gesellschaften zu fördern,

- die Interaktion aller in Palliative Care tätigen Berufsgruppen in der täglichen Praxis zu stärken,

- die wissenschaftliche Basis der einzelnen Betreuungsfelder zu verbessern,

- der Erarbeitung von Standards für die Ausbildung in Palliative Care im medizinischen und anderen Bereichen,

- die Fort- und Weiterbildung in Palliative Care zu unterstützen,

- Empfehlungen und Leitlinien für die Diagnostik und Therapie herauszugeben sowie Empfehlungen, Richtlinien und Stellungnahmen zu anderen das Fachgebiet betreffenden Fragen und Themen abzugeben,

- die Anliegen der Palliative Care in der Öffentlichkeit darzustellen.

\section{Ausbildung in Palliativmedizin}

Zunehmend werden in der universitären medizinischen Ausbildung palliativmedizinische Themen angesprochen. Die post-promotionelle Ausbildung in Palliativmedizin ist derzeit dadurch gekennzeichnet, dass sie zwar an den 28 Palliativstationen in Österreich möglich ist und auch stattfindet. Sie schlägt sich aber derzeit nicht strukturierterweise, etwa in einer eigenen Fach- oder Zusatzfachausbildung nieder, die die OPG anstrebt.

Die OPG bietet deshalb in Kooperation mit der Österreichischen Ärztekammer einen berufsbegleitenden Diplomlehrgang für Palliativmedizin an, der sich über ein Jahr erstreckt. Jährlich erwerben etwa vierzig Kollegen das entsprechende Diplom.

Univ.-Prof. Dr. Herbert Watzke Präsident 